\title{
Computed Tomographic Evaluation of Mid-thoracic Vertebral Corridors in Normal French Bulldogs
}

\author{
Adam Eby, Peter Early, Simon Roe, Karl Kraus, Lingnan Yuan, Jonathan Mochel
}

\section{ABSTRACT}

Objectives: (1) To report internal measurements of thoracic vertebral bone
morphology and (2) identify safe and clinically applicable surgical implant
corridors in the T7-T9 thoracic vertebrae of French Bulldogs.

Study Design: Observational, cross-sectional, descriptive study.

Sample Population: Seven client-owned French Bulldogs with normal thoracic vertebrae.

Methods: Computed tomographic (CT) studies of normal French Bulldogs
were reviewed. Multiplanar reconstruction of the CT images was used to
determine thoracic vertebral corridors. Corridor measurements included
the width, length, insertion distance off midline, and angle off midline
(sagittal) for each thoracic vertebra. One-way analysis of variance was
used to detect differences between groups.

Results: Measurements of vertebral corridor width $(\mathrm{p}>0.9848)$, length $(p>0.8113)$, implant center $(p>0.9282)$ and angle $(p>0.3609)$ did not differ between each vertebra. The average vertebral corridor width was $4.5 \pm 0.7$ $\mathrm{mm}$. The average corridor length was $17.2 \pm 2.5 \mathrm{~mm}$. The average corridor angle was $22.3 \pm 1.9^{\circ}$. The mean distance the proposed implant center was from the vertebral midline was $8.2 \pm 1.1 \mathrm{~mm}$. Inter-observer agreement of corridor length and implant center was good but poor for corridor angle and width.

Conclusion: Based on average corridor width and length, commercially available cortical screws or pins can be utilized for implants in this region of the thoracic spine. The angle of corridor trajectory from a dorsal approach seems most applicable for T7-T9.

Clinical Significance: Vertebral corridors can be measured using CTMPR, and implant specifications and angles derived. Surgical guides can also be created to guide implant placement. This approach provides a simple and accurate method to guide the placement of thoracic vertebral implants.

Keywords: Corridors, dog, thoracic, vertebrae.
Published Online: February 1, 2022

ISSN: $2736-6596$

DOI : $10.24018 /$ ejvetmed.2022.2.1.21

\section{A. Eby}

Department of Clinical Sciences, College of Veterinary Medicine, NC State University, Raleigh, NC, USA.

(e-mail: aeby@ncsu.edu)

P. Early*

Department of Clinical Sciences, College of Veterinary Medicine, NC State University, Raleigh, NC, USA.

(e-mail: pjearly@ncsu.edu)

\section{S. Roe}

Department of Clinical Sciences, College of Veterinary Medicine, NC State University, Raleigh, NC, USA.

(e-mail: sroe@ncsu.edu)

K. Kraus

Department of Clinical Sciences, College of Veterinary Medicine, Iowa State University, Ames, IA, USA.

(e-mail: kkraus1@iastate.edu)

L. Yuan

Department of Clinical Sciences, College of Veterinary Medicine, Iowa State University, Ames, IA, USA.

(e-mail: lynn94@iastate.edu) J. Mochel

Department of Clinical Sciences, College of Veterinary Medicine, Iowa State University, Ames, IA, USA.

(e-mail: jmochel@iastate.edu)

*Corresponding Author

\section{INTRODUCTION}

Complex musculoskeletal deformities occur in the axial or appendicular skeleton and may contribute to significant pain and disability if left untreated. Malformations within the axial skeleton, specifically involving the thoracic vertebrae, are common among the canine population, increasing frequency among brachycephalic dog breeds such as French bulldogs, English bulldogs, Boston terriers, and Pugs. Various malformations have been described, with malformation of the vertebral body a common finding. These malformed vertebrae are often located in the mid to caudal thoracic vertebral region, usually between $\mathrm{T} 7$ and $\mathrm{T} 9$, with $\mathrm{T} 7$ and $\mathrm{T} 8$ most commonly affected [1]-[4].

Vertebral malformations disturb normal vertebral column anatomy and biomechanics, leading to deformity of the vertebral column and possible neurological dysfunction. Surgical treatment via decompression or stabilization techniques is often implemented to improve or alleviate such neurological dysfunction. Placement of surgical implants within the thoracic spine is usually performed via a dorsal surgical approach. This requires both an in-depth understanding of vertebral anatomy as well as precise spatial awareness to guide and place surgical implants successfully.

Proposed canine vertebral corridors using computed tomography were recently described by [5]. The length and width of the corridor were found most variable of measurements and were largely dependent on dog size. The corridor angle was not altered by breed or size of the dog, and from T5 to T9, it was similar at 23 degrees from the midsagittal plane of vertebra across all dogs. More variability was noted in the cranial thoracic spine at $\mathrm{T} 1$ to $\mathrm{T} 3$, though the 
corridor angle was not different from T5 to T9. Thoracic corridor dimensions were also found to be amendable to commercially available cortical screws and pins in dogs weighing 3.1 to 60 kilograms.

To the authors' knowledge, mid-thoracic vertebral corridors have not been described nor defined in the normal French Bulldog. Therefore, the objective of this study was to report internal measurements of thoracic vertebral bone morphology and identify safe and clinically applicable surgical implant corridors in the T7-T9 thoracic vertebrae of normal French Bulldogs from a dorsal approach. We hypothesized that vertebral corridors in normal French Bulldogs would be similar throughout the mid-thoracic spine, similar to vertebral corridors of other normal canine spines, and of the suitable size to accommodate commercially available implants.

\section{MATERIALS AND METHODS}

\section{A. Inclusion Criteria}

Medical and imaging databases were searched for clientowned dogs that underwent CT scans at NC State University CVM between January 2003 and December 2019 (n=53) and Iowa State University CVM between January 2007 and December $2019 \quad(n=20)$. Inclusion criteria consisted of skeletally mature ( $>12$ months of age) French Bulldogs with diagnostic imaging studies in which the complete thoracic spine was included and vertebral segments T7-T9 were considered normal by the reporting radiologist and by the authors of this study. Exclusion criteria included significant pathology, presence of implants, or previous history of fractures involving vertebral segments T7-T9. Significant pathology was defined as any hypoplastic vertebrae, hemivertebrae, or other vertebral body malformations, intervertebral disc protrusion or extrusion, any evidence of spondylosis deformans, or evidence suggestive of neoplasia of the vertebral column. Seven patients met the inclusion criteria and were further analyzed.

\section{B. Image Acquisition}

Scans were acquired using a Siemens SOMATOM Perspective 64 Multislice CT Scanner (Siemens Medical Solutions, Malvern, Pennsylvania) or a 16 slice Canon Aquilion scanner (TXX201A, Canon Medical Systems, Tustin, California). Scan settings included: helical acquisition, 1 to 2 -mm slice thickness, and were $512 \times 512$ pixels in size, $\mathrm{kVp} 120$, mAs 300 , with a median field of view of $24 \mathrm{~cm}$ (range 24-50). All scans were performed in a cranial to caudal direction, and images were reconstructed using a bone algorithm.

\section{Measurements}

Image analysis of the CT data was performed with a free open source DICOM viewer (Horos V3.3.6, OsiriX, horosproject.org). Measurements were performed by using a three-dimensional multiplanar reconstruction of each CT dataset. All measurements were performed within the 2D transverse viewpoint. The frontal and sagittal planes were aligned with the long axis of the thoracic vertebrae T7-T9. The frontal plane was aligned using the center of the spinal canal as midline. The sagittal plane was aligned using a line of best fit along the dorsal aspect of vertebral bodies T7-T9. The transverse plane was then manipulated along the cranial aspect of each vertebra until visualization of both vertebral pedicles (right and left) was observed (see Fig. 1).

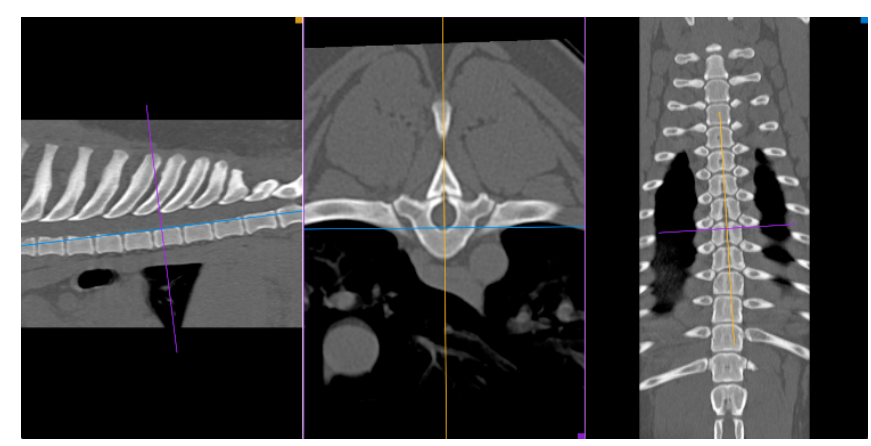

Fig. 1. A multiplanar reconstruction view of T8, with sagittal, transverse, and dorsal planes. Imaging planes were manipulated to obtain a true transverse image of the desired vertebra to be measured. The sagittal plane was aligned with the ventral aspect of the spinal canal. The dorsal plane was aligned with the midline bisecting the spinous process, spinal cord, and vertebral body.

Transverse cross-sections through each thoracic vertebrae (T7-T9) were then analyzed. A series of measurements for each cross-section were manually obtained. Measurements performed included corridor width and length, angle of trajectory, and implant center. The width was the narrowest point across the corridor. The length was the longest point extending from the proposed insertion point at the cis cortex extending into the trans cortex of the vertebral body. The angle of the trajectory was assessed in relation to the midsagittal plane of the spinous process. The implant center was the distance from the mid-sagittal plane to the implant insertion center (see Fig. 2).

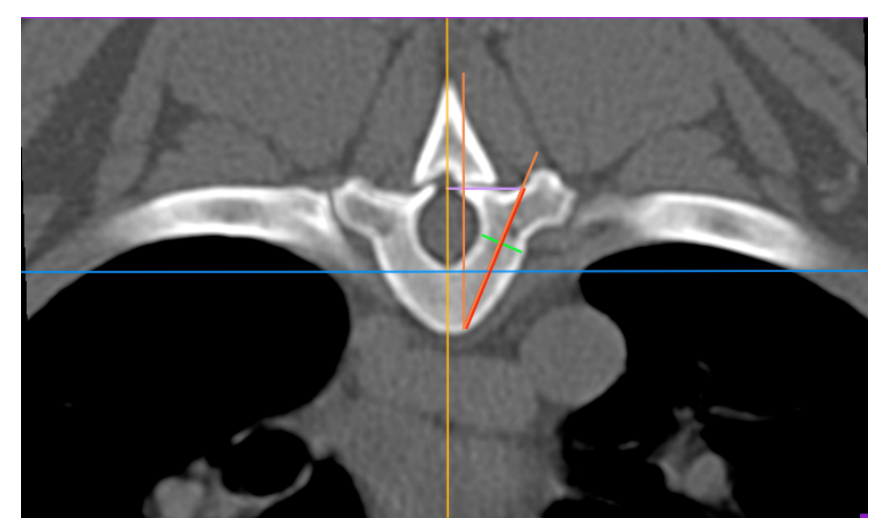

Fig. 2. Transverse computed tomography (CT) image through the vertebral body of T8 in a bone window. This image is representative of measurements obtained. Measurements performed included corridor width (yellow) and length (red), angle of the trajectory (orange), and implant center (purple).

Three independent observers performed measurements: one American College of Veterinary Internal Medicine (ACVIM) board-certified, surgically trained neurologist, one American College of Veterinary Surgeon (ACVS) boardcertified surgeon, and one ACVS surgically trained resident. Minimal instruction in the form of planar alignment and proposed corridors was provided to each observer performing the measurements to avoid bias within the data set. For 
proposed corridors, instruction was provided to measure the pedicle and vertebral body, resulting in the most significant amount of bone purchase for proposed implant placement from a dorsal approach. There was no standardization of the proposed corridor for the observers and a standard bone window was utilized for all measurements performed.

Data Analysis

Vertebral measurements of length, width, angle, and implant insertion were collected and analyzed to determine the mean and standard deviations. A Tukey's Honest Significant Difference (HSD) test was performed to assess average corridor angle differences between each thoracic vertebra. Interobserver agreements were measured by the intraclass correlation coefficient (ICC). All analyses were conducted in R 3.5.2 (R Core Team 2017, Vienna, Austria, https://www.R-project.org), and $\mathrm{p}<0.05$ was considered significant.

\section{RESULtS}

CT studies containing the 73 thoracic spines of French Bulldogs were performed during the study period. After applying exclusion criteria, a total of seven studies were included. The mean age and body weight was $5.5 \pm 4.7$ years and $12.3 \pm 4.0 \mathrm{~kg}$, respectively. There were five neutered males and two intact males. The mean corridor width was 4.5 $\pm 0.7 \mathrm{~mm}$. The mean corridor length was $17.2 \pm 2.5 \mathrm{~mm}$. The mean corridor angle was $22.3 \pm 1.9^{\circ}$. The proposed implant center's mean distance from the vertebral midline was $8.2 \pm$ $1.1 \mathrm{~mm}$.

\section{Discussion}

This study evaluated mid-thoracic vertebral corridors (T7T9) in normal French Bulldogs using CT MPR. In line with our hypothesis, our results revealed mid-thoracic vertebral corridors to be similar throughout. Additionally, based on measurements obtained in this study, commercially available sizes of cortical screws and positive profile pins would be suitable for placement in normal T7-T9 vertebrae in French Bulldogs. Common fixation methods within the thoracic spine include pins, surgical screws, or bone plates. The use of pins and non-locking screw constructs allows for more degrees of freedom in placement within an implant corridor. In this study, the corridor orientation was proposed from a dorsal surgical approach. The corridor length and width were similar throughout each thoracic vertebrae and did not differ within the French Bulldog breed. The most clinically helpful measurement obtained was the angle of the corridor orientation. The angle was not altered significantly within the French Bulldog breed nor differed among the different vertebrae measured. The angle of the corridor from T7-T9 was statistically similar and measured approximately 22 degrees across all dogs in the sample.

Thoracic vertebral corridor measurements in normal French Bulldogs were also found to be similar to normal dogs of various breeds and sizes [5]. The corridor angle within this study was not altered by breed or size of dog from $\mathrm{T} 5$ to $\mathrm{T} 9$ and was similar at 23 degrees across all dogs. This finding resembled those from our study, where the corridor angle was not altered significantly within the French Bulldog or between the vertebrae measured and was similar at 22 degrees across all dogs. While direct comparison of the two studies is not possible due to variations in study design, both studies were similar regarding a patient population (normal spines), study design, and vertebral measurements performed. Further evaluation in a more controlled environment or inclusive study is warranted to allow for a more direct comparison.

Corridor angle may be the most clinically reliable measurement when comparing thoracic vertebral corridors across dog breeds. Measurement of CT vertebral corridors preoperatively is recommended and critical to helping guide surgical implant placement in a patient-specific manner. Overall, mid-thoracic corridor dimensions are similar in most dog breeds and amendable to commercially available cortical screws and pins.

There are a few limitations that should be considered. Mainly, clinically normal mid-thoracic vertebrae were utilized, limiting the application to dogs with significant vertebral pathology. Furthermore, clinically normal thoracic spines in the French Bulldog were uncommon given the inclusion criteria and accounted for less than $10 \%$ of patients identified at various academic institutions. Multiple independent observers were utilized to perform measurements and manipulate study images, resulting in variability in their interpretation and reduced statistical significance for some measurements.

\section{CONCLUSION}

In conclusion, the present study is the first to assess midthoracic vertebral corridors (T7-T9) in normal French Bulldogs. Measurements performed using CT MPR revealed mid-thoracic vertebral corridors to be similar throughout, which are amendable to commercially available sizes of cortical screws, and positive profile pins would be suitable for placement in normal T7-T9 vertebrae in French Bulldogs.

\section{CONFLICT OF INTEREST}

The authors declare that they do not have any conflict of interest.

\section{REFERENCES}

[1] Bailey CS, Morgan JP. Congenital spinal malformations. Vet Clin North Am Small Anim Pract. 1992; 22(4): 985-1015.

[2] Westworth DR, Struges BK. Congenital spinal malformations in small animals. Vet Clin North AmSmall Anim Pract. 2010; 40(5): 951-981.

[3] Moissonnier P, Gossot P, Scotti S. Thoracic kyphosis associated with hemivertebra. Vet Surg. 2011; 40(8): 1029-32.

[4] Gutierrez-Quintana R, Guevar J, Stalin C, Faller K, Yeamans C, Penderis J. A proposed radiographic classification scheme for congenital thoracic vertebral malformations in brachycephalic "screwtailed" dog breeds. Vet Radiol Ultrasound. 2014; 55(6): 585-91.

[5] Schmitt EM, Early P, Bergman R, et al. Computed tomography evaluation of proposed implant corridors in canine thoracic vertebrae. Vet Surg. 2021; 50(7): 1427-1433. 